DOI: http://dx.doi.org/10.18569/tempus.v14i1.2655

\title{
Fluoteração das águas de abastecimento público no estado de pernambuco: do estado da arte à vigilância da saúde bucal
}

\section{Fluoridation of the waters of public supply in the state of pernambuco: the state of the art and the vigilance of oral healthe}

\section{Fluoteración de aguas de suministro público en el estado de pernambuco: del estado del arte a la vigilancia de la salud}

\author{
Clébia Roberta Eufrazio do Nascimento \\ Brenda Rocha Borba de Andrade ${ }^{1}$ \\ José Alison Baracho da Silva ${ }^{2}$ \\ Petrônio José de Lima Martelli ${ }^{3}$
}

RESUMO: A cárie dentária representa, em termos de saúde bucal, o principal agravo em saúde pública no Brasil, atingindo, ainda que de modo desigual, grande parte da população brasileira. $\mathrm{O}$ uso do flúor em saúde pública, sob a forma de fluoreto, é considerado o principal fator de proteção, decisivo para a obtenção de expressiva redução na prevalência da doença. A fluoretação das águas é considerada a principal medida para reduzir a prevalência da doença. Apesar da fluoretação ser obrigatória no Brasil desde 1974, várias cidades brasileiras não fluoretam suas águas. O objetivo deste estudo foi analisar teores de flúor nas águas de abastecimento público da $3^{\text {a }}$ macrorregião de saúde de Pernambuco, em municípios com 50.000 habitantes ou mais. Trata-se de uma pesquisa de estudo analítico transversal a partir da comparação obtida pelos dados primários do projeto VIGIFLÚOR e secundários disponibilizados pela COMPESA,Sistema de Informação de Vigilância da Qualidade da Água para Consumo Humano (SISÁGUA), do Instituto Brasileiro de Geografia e Estatística (IBGE) e do Sistema Nacional de Informação sobre Saneamento (SNIS). Foi observado a presença de flúor natural em teores significativos nos mananciais, porém essa concentração não chega na ponta da rede de abastecimento público. Este estudo reforça a necessidade de que a água de abastecimento dos municípios de Serra Talhada, Arcoverde e Buíque sejam fluoretados artificialmente e o seu controle de vigilância seja realizado por meio de programas de heterocontrole, para atingir seu benefício máximo para proteção da cárie e evitar o risco de causar fluorose.

PALAVRAS CHAVES: Fluoretação, Cárie dentária, SUS

1 Cirurgiã-dentista graduada pela UFPE

2 Graduado em Geografia pela UFPE, graduando em Geologia pela UFPE

3 Professor Adjunto da Área Acadêmica de Medicina Social - UFPE

ISSN 1982-8829 Tempus, actas de saúde colet, Brasília, 14(1), 77-88, mar, 2020. Epub Mai/2020 
ABSTRACT: The dental cavity representsen terms of oral health, the main lamafe in public health in Brazil, reacleing thongh in anunequal way, a great part of the Brazilian population. The use offl uorine in public health under the formof fluorideis considered the major factor of protection, decisive for the attainmentofan expressive reduction in theprevalenceofthedisease. Al though fluoridation has heen compulsory since 1974 in Brazil, several Brazilian town sdon't fluoridate their waters as is the case of the muncipality in the state of Pernambuco. The ainofthis study was to analyze the proportion off luoride in the waters of public supply of the third health macroregion of Pernambuco, in towns with 50.000 in habitants more. It refersto a researdeof a crossan alyticalstudy from the comparison obtained by the secondary data forwarded by "COMPESA", by the primary data of the project "VIGIFLÚOR", system of information of vigilance of the water quality for human consume (SISÁGUA), data of the Brazilian Institute of Geograph and Statistics (IBGE) andthenational system of information about samitation (SNIS). It wasobservedthepresenceof natural fluoride in a meaningfulproportion in thefountain'tget to thetipof the net of publicheads, butthisconcentrationdoesn'tsupply. This study high lights the need for the water supply of thet owns of Serra Talhada, Arcoverde and Buíque to be fluoridated artificially and the ircontrol of vigilance to be carried out byprogramsofheterocontrol in ordertoreachtheirmaximumbenefits for theprotectionagainstcavities anda theriskofcausingfluorosis.

KEY WORDS:Fluoredation, Caries dental, Unified Heath System

RESUMEN: La caries dental representa, en términos de salud bucal, el principal problema de salud pública en Brasil, que afecta, aunque de manera desigual, a una gran parte de lapoblación brasileña.El uso de flúor enlasalud pública en forma de flúor se considera el principal factor de protección, decisivo para obtener una reducción significativa enla prevalencia de laenfermedad. La fluoracióndel agua se considera la medida principal para reducirla prevalencia de laenfermedad. Aunquelafluoraciónha sido obligatoriaen Brasil desde 1974, variasciudadesbrasileñas no hanfluorizado sus aguas. El objetivo de este estudiofueanalizarelcontenido de fluoruroenelsuministro público de agua de la 3ra macro región sanitaria de Pernambuco, enmunicipios con 50,000 habitantes o más. Este es unestudio analítico transversal basadoen una comparación de datosprimariosdelproyecto VIGIFLÚOR y datossecundarios proporcionados por COMPESA, el Sistema de Informaciónde Vigilancia de la Calidad del Agua para el Consumo Humano (SISÁGUA) del Instituto Brasileño de Geografía y Estadística. (IBGE) y el Sistema Nacional de Información sobre Saneamiento (SNIS). Se observóla presencia de fluoruro natural en niveles significativos enlosmanantiales, pero esta concentración no llega al final de lared de suministro público. Este estudiorefuerzalanecesidad de unsuministro de agua fluorada artificialmente enlosmunicipios de Serra Talhada, Arcoverde y Buíque y sucontrol de vigilancia se llevará a cabo a través de programas de heterocontrol para lograr su máximo beneficio para laprotección de la caries y prevenir riesgo de causar fluorosis.

PALABRAS CLAVE:Fluoredation, Dental caries, Sistema Único de Salied 


\section{INTRODUÇÃO}

A cárie dentária representa, no escopo da saúde bucal, um dos principais agravos em saúde pública no Brasil, atingindo, ainda que de modo desigual, indivíduos de todas as faixas etárias e diferentes níveis socioeconômicos ${ }^{1}$.

Desde meados do século XX, mantendo-se como tendência na primeira década do século XXI, estudos de base epidemiológica registram declínios da prevalência de cárie nos países industrializados e em alguns países em desenvolvimento ${ }^{2}$. O uso do flúor, sob a forma de fluoreto, é considerado o principal fator de proteção, sendo decisivo para a obtenção dessa expressiva redução na prevalência da doença. De maneira geral, os principais meios utilizados nas estratégias populacionais de uso de fluoretos para prevenir a cárie são o creme dental e a água de abastecimento público.

A fluoretação dos sistemas públicos de abastecimento é considerada a medida de saúde mais eficaz na prevenção da doença, por apresentar grande alcance populacional, ser considerada segura, benéfica à saúde bucal, isenta de efeito colateral, e não exigir esforço dos indivíduos ${ }^{2}$.

Porém, desde sua implantação, a despeito de sua comprovada eficácia, segurança e custoefetividade, a fluoretação das águas tem enfrentado oposição de alguns grupos, que utilizam como principais argumentos: violação do princípio ético da autonomia; questionamentos sobre a efetividade do método; e a possibilidade de ocasionar riscos para a saúde, como a fluorose dentária devido à ingestão excessiva do flúor durante a fase de formação do dente.

O Centro de Controle e Prevenção de Doenças (CDC), dos Estados Unidos, admite que o poder preventivo da água fluoretada é de $40 \%$ a $70 \%$, em crianças, dependendo do índice de prevalência de cárie, reduzindo também a perda dos dentes em adultos entre $4 \%$ a $60 \%$ sendo considerada uma das dez mais importantes medidas de saúde pública do século $\mathrm{XX}^{2,3}$.

Além do CDC, a Organização Mundial da Saúde (OMS) reconhece e indica a fluoretação como medida de saúde pública, porém para que a população se beneficie dos efeitos dessa importante medida, faz-se necessário que os teores adicionados à água estejam dentro dos níveis recomendados 2,4 .

É descrita, portanto, como uma medida de atenção primária em saúde bucal, que utilizada como um procedimento profilático-preventivo de controle da cárie, pode propiciar a inibição e o tratamento de estágios iniciais da doença quando houver contínua exposição de fluoretos na cavidade bucal, o que sugere que o efeito da substância adicionada à água não é apenas sistêmico, mas deve atuar também localmente no esmalte dentário ${ }^{3,5}$.

No Brasil, a fluoretação das águas é obrigatória desde 1974, regulamentada pela Lei Federal $n^{\text {o }} 6050$ (BRASIL, 1974) ${ }^{6}$, entretanto a cobertura da fluoretação nos municípios tem sido pouco documentada, tornando obscuro o conhecimento sobre o acesso ao benefício nas diversas localidades

ISSN 1982-8829 Tempus, actas de saúde colet, Brasília, 14(1), 77-88, mar, 2020. Epub Mai/2020 
do território brasileiro, mesmo sendo a ampliação deste acesso uma das prioridades da Política Nacional de Saúde Bucal (PNSB) ${ }^{9}$. Dados do levantamento de saúde bucal realizado no Brasil em 2003, revelam que 115 (46\%) dos 250 municípios avaliados proporcionavam acesso à água fluoretada. As regiões Sudeste e Sul eram as que estavam em melhor situação quanto à cobertura, com $66 \%$ e $88 \%$, respectivamente ${ }^{1,7,9,8}$.

Em 2003, a fluoretação das águas de abastecimento público, atingia uma população de aproximadamente 70 milhões de brasileiros. Informações do Ministério da Saúde (MS) ${ }^{9}$ apontam que até o mês de junho de 2005 foram implantados 121 novos sistemas de fluoretação de águas, abrangendo seis estados e beneficiando cerca de 500 mil pessoas. Ainda segundo o MS, outros 430 projetos técnicos foram submetidos a análises, o que beneficiaria cerca de seis milhões de brasileiros. A viabilização destes projetos está sendo concretizada por meio de ação conjunta entre a Fundação Nacional de Saúde (FUNASA) e convênios com as Secretarias Estaduais de Saúde ${ }^{8}$.

O estado de Pernambuco, que permanece atualmente sem uma política de fluoretação de águas, apesar de alguns municípios terem fluoretado suas águas em diferentes momentos históricos, foi um dos estados que submeteram um projeto afim de viabilizar tal política pública, sendo este aprovado pela câmara técnica do MS. Sendo assim, a Secretaria Estadual de Saúde (SES) está em processo de implantação de um sistema de fluoretação, com previsão de início para o ano de 2014, mas não conseguindo até o início de 2019 realizar a sua operacionalização.

Enormes são as expectativas de que esse projeto consiga de fato ser implantado e impacte positivamente a condição de saúde bucal de toda população pernambucana, pois até o presente momento, poucos municípios do estado tiveram suas águas fluoretadas conforme preconiza a legislação nacional.

Com a implantação do sistema de fluoretação das águas no estado de Pernambuco se faz necessário, dentre outras medidas, o conhecimento prévio da realidade encontrada por meio de um resgate histórico na literatura científica e em documentos públicos, além da avaliação das águas para verificar a presença de flúor natural e/ou artificial, afim de contribuir para a instrumentalização das instâncias de gestão do Sistema Único de Saúde (SUS) com dados indispensáveis para a definição de estratégias.

Dessa forma, este estudo analisa a problemática do acesso à água fluoretada na $3^{\mathrm{a}}$ macrorregião de saúde do Estado de Pernambuco, verificando em mananciais e nas redes de abastecimento públicas de água, as concentrações adequadas e inadequadas dos fluoretos.

\section{METODOLOGIA DO TRABALHO}

Trata-se de estudo descritivo a partir da comparação de dados da COMPESA, do projeto VIGIFLÚOR $^{4}$,edoSistema de Informação de Vigilância da Qualidade da Água para Consumo

4 Estudo multicêntrico voltado à descrição da cobertura populacional da fluoretação das águas da rede pública de Tempus, actas de saúde colet, Brasília, 14(1), 77-88, mar, 2020. Epub Mai/2020 ISSN 1982-8829 


\section{$81 / /$}

Humano (SISAGUA), sobre a fluoretação de águas em municípios maiores de 50.000 habitantes na $3^{a}$ macrorregião de saúde de Pernambuco, e de dados do Instituto Brasileiro de Geografia e Estatística (IBGE) e do Sistema Nacional de Informação sobre Saneamento (SNIS) a respeito da população residente nos municípios e com acesso a água tratada, respectivamente.

O estado de Pernambuco é o sétimo estado mais populoso do Brasil, com 8.796.032 habitantes, o que corresponde a aproximadamente $4,6 \%$ da população brasileira, distribuídos em 185 municípios. Esses municípios por sua vez, estão divididos em quatro macrorregiões.

Escolheu-se, por amostra de conveniência, a $3^{\mathrm{a}}$ macrorregião (Figura 1), composta pela $6^{\mathrm{a}}, 10^{\mathrm{a}}$ e $11^{\text {a }}$ Gerências Regionais de Saúde (GERES). Tal escolha se deu pela disponibilidade dos laudos de teores de flúor tanto da COMPESA quanto do projeto VIGIFLÚOR. Os municípios estudados foram municípios pertencentes a $6^{\mathrm{a}}$ e $11^{\mathrm{a}}$ GERES, respectivamente, com mais de 50.000 habitantes - Arcoverde, Buíque (6 ${ }^{\mathrm{a}}$ GERES) e Serra Talhada (11 ${ }^{\mathrm{a}}$ GERES).

\section{Figura 1 - Mapa das quatro macrorregiões do estado de Pernambuco.}

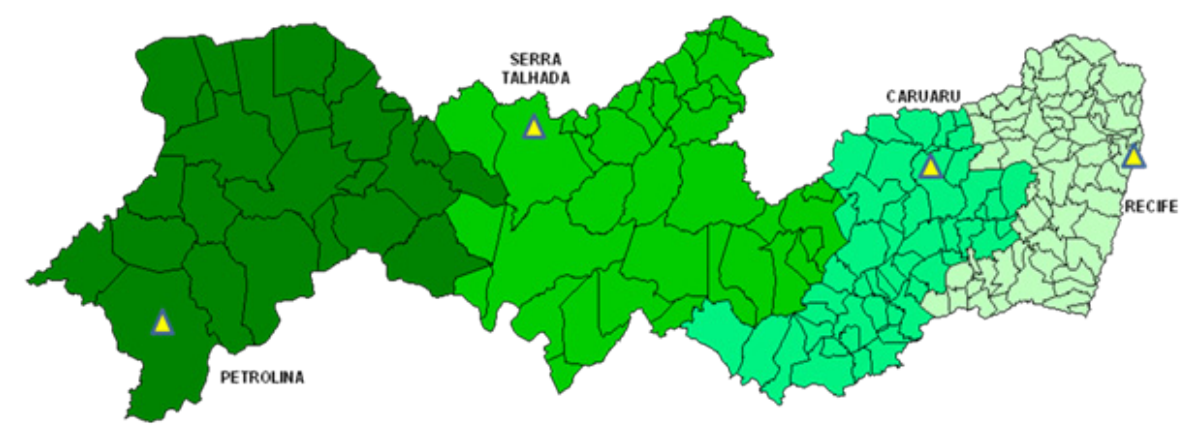

\section{$3^{\mathrm{a}}$ Macrorregião}

A coleta de dados do projeto VIGIFLÚOR (amostras de água) foi realizada por meio de servidores públicos vinculados ao Sistema Único de Saúde (SUS), com atuação no setor de vigilância em saúde, os quais foram previamente calibrados pelos pesquisadores e denominados agentes Vigiflúor.

Para a realização dessa coleta foi adotado o estabelecimento de duas amostras de água principais localizadas em unidades distintas por estação de tratamento ou solução alternativa existente em cada município e complementada por outras duas amostras de controle também localizadas em unidades distintas uma para cada amostra principal. Por esse critério, admite-se não haver redução dos níveis agregados nas estações de tratamento ao longo da rede de distribuição, mantendo-se a estabilidade dos teores do flúor até os pontos mais distantes, e que não tenha sido identificado

abastecimento em municípios com mais de 50 mil habitantes, na primeira metade da segunda década do século XXI. Pretende também identificar as localidades que vêm desenvolvendo atividades de vigilância dessa medida em âmbito municipal e estadual. De modo complementar será analisada, laboratorialmente, a concentração de fluoreto em localidades selecionadas, para confirmar ou conhecer o teor do halogênio presente em águas de abastecimento público para consumo humano.

ISSN 1982-8829 Tempus, actas de saúde colet, Brasília, 14(1), 77-88, mar, 2020. Epub Mai/2020 
pontos de fragilidade ou de mistura de águas no município.

Os pontos de coleta das amostras principais foram escolhidos preferencialmente em unidades públicas da área da educação ou da saúde, em ponto da rede mais próximo da unidade de tratamento (estação de tratamento de água, reservatório ou solução alternativa) e nas unidades públicas da área da educação ou da saúde em ponto da rede mais distante da unidade de tratamento (estação de tratamento de água, reservatório ou solução alternativa).

As amostras de água foram coletadas durante três meses consecutivos, sempre no mesmo ponto de coleta, totalizando 12 amostras por estação de tratamento. A frequência da coleta foi mensal e todas realizadas no mesmo dia, apenas alternando as datas de coleta a cada mês. Depois de coletadas as amostras foram enviadas para o laboratório de Bioquímica da Faculdade de Odontologia de Piracicaba da Universidade Estadual de Campinas (FOP-Unicamp), e o teor de fluoreto foi aferido por meio de método eletrométrico.

Os dados secundários provenientes de laudos da COMPESA, são dados de controle de mananciais que a mesma executa regularmente, disponibilizados em formato digital para essa pesquisa. Ou seja, tais dados são referentes a quantidade de flúor natural detectados nas águas de mananciais, e não na rede de abastecimento público. Os valores utilizados como referência para análise de teores de flúor, tanto dos laudos da COMPESA quanto do Projeto VIGIFLÚOR, foram os valores mínimos e máximos obtidos nos laudos que nos foram disponibilizados.

OSISAGUA, outro sistema que foi utilizado para captura de dados secundários para essa pesquisa, é uma ferramenta de gestão do Programa Nacional de Vigilância da Qualidade da Água para Consumo Humano (VIGIÁGUA) ${ }^{11}$, e tem por objetivo sistematizar dados de qualidade da água dos estados, municípios e distrito federal, e gerar relatórios, de forma a produzir informações necessárias à prática da vigilância.

Levando em consideração a relação concentração de fluoreto e variação média das temperaturas máximas diárias do ar de cada região, foi estabelecido que a concentração de fluoreto nas águas que oferece o benefício máximo para cárie dentária e risco mínimo para fluorose, para as localidades selecionadas pela pesquisa, cujas médias das temperaturas máximas diárias do ar se situem entre $26,3^{\circ} \mathrm{C}$ e $32,5^{\circ} \mathrm{C}$ oscilam entre 0,55 a $0,84 \mathrm{mgF} /$ Lconforme descrito no Quadro 1. 
Quadro I - Consenso sobre o benefício máximo e risco mínimo para teores de flúor na água de abastecimento público em temperaturas médias anuais entre $26,3^{\circ} \mathrm{C}$ e $32,5^{\circ} \mathrm{C}$.

\begin{tabular}{|c|c|c|}
\hline $\begin{array}{c}\text { TEOR DE FLÚOR NA } \\
\text { ÁGUA } \\
(\mathrm{em} \mathrm{PPM} \mathrm{ou} \mathrm{mgF/L)}\end{array}$ & $\begin{array}{c}\text { BENEFÍCIO } \\
\text { (prevenir cárie) }\end{array}$ & $\begin{array}{c}\text { RISCO } \\
\text { (produzir fluorose dentária) }\end{array}$ \\
\hline 0,00 a 0,44 & Insignificante & Insignificante \\
\hline 0,45 a 0,54 & Mínimo & Baixo \\
\hline 0,55 a $0,84\left(^{*}\right)$ & Máximo & Moderado \\
\hline 0,85 a 1,14 & Máximo & Alto \\
\hline 1,15 a 1,44 & Questionável & Muito Alto \\
\hline 1,45 ou mais & Maleficio & \\
\hline
\end{tabular}

Sendo assim, utilizou-se tais valores referenciais para analisarmos os riscos e benefícios do flúor nas águas dos municípios estudados (Arcoverde, Buíque e Serra Talhada).

Este estudo está vinculado ao projeto "Cobertura e vigilância da fluoretação da água de abastecimento público no Brasil", vinculado ao Centro Colaborador do Ministério da Saúde da Universidade de São Paulo (CECOL/USP), e foi devidamente aprovado pelo Comitê de Ética em Pesquisa da FSP-USP sob o protocolo de pesquisa $n^{\circ} 22186513.8 .0000 .542$.

\section{RESULTADOS}

O guia da Agência Nacional de Águas (ANA) ${ }^{16}$ define que para os municípios abrangidos na pesquisa o abastecimento de águas é feito pelos seguintes mananciais: Arcoverde, localizado com divisa ao Agreste, integrante da mesorregião do Sertão Pernambucano e pertencente à microrregião do Sertão do Moxotó, está inserido nos domínios das bacias hidrográficas dos rios Ipanema e Moxotó, contando ainda com açudes municipais, dentre eles o açude Riacho do Pau; Buíque, localizado no Agreste Meridional e Planalto da Borborema, está incluído na abrangência do semiárido tendo alto índice de aridez e risco de seca, sendo abastecido nos domínios da bacia hidrográfica do Rio Ipanema sendo utilizado também o Açude Mulungu; Serra Talhada, localizada no Sertão do Pajeú conta com o Açude Cachoeira II sendo da região do Vale do Pajéu, abastecido nos domínios da bacia hidrográfica do Rio Pajéu, ambos localizados no Sertão Central, como pode ser observado no Quadro II. Esses mananciais serviram para análise dos teores de flúor que abastecem cada região. 
Quadro II - Cobertura populacional e população urbana com acesso à água tratada dos municípios da $3^{\mathrm{a}}$ macro região de saúde, do estado de Pernambuco, com mais de 50.000 habitantes.

\begin{tabular}{|c|c|c|c|}
\hline MUNICÍPIO & $\begin{array}{c}\text { POPULAÇÃO } \\
\text { TOTAL } \\
(\text { IBGE/2016) }\end{array}$ & $\begin{array}{c}\text { POPULAÇÃO } \\
\text { URBANA COM } \\
\text { ACESSO À ÁGUA DE } \\
\text { ABASTECIMENTO } \\
(\text { SNIS/2015) }\end{array}$ & $\begin{array}{c}\text { PORCENTAGEM (\%) } \\
\text { DA POPULAÇAO COM } \\
\text { ACESSO À ÁGUA DE } \\
\text { ABASTECIMENTO }\end{array}$ \\
\hline ARCOVERDE & 73.154 & 67.787 & $92,66 \%$ \\
\hline BUÍQUE & 57.120 & 17.066 & $29,88 \%$ \\
\hline SERRA TALHADA & 84.970 & 84.352 & $99,27 \%$ \\
\hline & & & \\
\hline
\end{tabular}

1 Fonte: $\underline{\text { http://www.ibge.gov.br }}{ }^{17}$

2 Fonte: $\underline{\text { http://www.snis.gov.br/ } / 18}$

Dentre os três municípios com mais de 50.000 habitantes da $3^{\text {a }}$ macrorregião abrangidos pela pesquisa verificamos que, o município de Serra Talhada, é o que apresenta maior acesso à água tratada por parte da sua população com 99,27 \%; seguido pelo município de Arcoverde com 92,66 $\%$; e Buíque com $29,88 \%$ conforme demonstra o quadro II.

Os dados fornecidos pelo SISAGUA 2008, demonstram que nenhum dos três municípios estudados possui cadastro no sistema sobre o controle e vigilância do teor de flúor nas suas águas.

Os dados de teores de flúor na pesquisa foram obtidos utilizando os valores máximos e mínimos disponibilizados tanto pela COMPESA quanto pelo VIGIFLÚOR, também descrito no quadro III.

Para o município de Arcoverde os valores de teores de flúor natural variaram entre 0,00 a 0,010, já o projeto VIGIFLÚOR mostrou outros valores que chegam à ponta da torneira da população, esses valores variaram entre 0,10 a 0,20 .

O município de Buíque foi considerado perda amostral pois não foram realizadas as coletas da amostra de água para análise laboratorial do fluoreto. Serra Talhada por sua vez, tem os mesmos valores de teores de flúor natural de acordo com a COMPESA 0,00 a 0,00 logo nos laudos realizados pela pesquisa mostrou valores insignificantes de flúor que chega a torneira da população, encontrados pelo projeto VIGIFLÚOR valores entre 0,12 a 0,14 .

\section{DISCUSSÃO}

Os dados do quadro III demonstram o quanto seria impactante que os municípios recebessem acréscimo de flúor em suas águas de abastecimento público, pois uma quantidade representativa de moradores receberiam os benefícios da fluoretação, já que, o nível de abrangência de cobertura 


\section{$85 / /$}

pode chegar a quase $80 \%$ em algumas cidades, como foi o caso de Serra Talhada.

Quadro III - Teores de flúor dos municípios da $3^{\text {a }}$ macro região de saúde, do estado de Pernambuco, com mais de 50.000 habitantes, segundo a COMPESA, e o VIGIFLUOR.

\begin{tabular}{|c|l|l|c|c|}
\hline $\begin{array}{l}\text { M A C R O } \\
\text { REGIÃO }\end{array}$ & MUNICÍPIOS & MANANCIAIS ${ }^{1}$ & $\begin{array}{c}\text { TEORES } \\
\text { DE FLÚOR } \\
\text { NATURAL DOS } \\
\text { MANANCIAIS } \\
\text { NATURAL(MG/ } \\
\text { Fl) }\end{array}$ & $\begin{array}{c}\text { TEORES DE } \\
\text { FLÚOR } \\
\text { LAUDO } \\
\text { UNICAMP (MG/ } \\
\text { Fl) }\end{array}$ \\
III & ARCOMPESA) & (VIGIFUU) $^{3}$ \\
\hline III & BUÍQQUE* & $\begin{array}{c}\text { Açude Riacho do } \\
\text { Pau }\end{array}$ & $0,00-0,010$ & $0,10-0,20$ \\
\hline III & $\begin{array}{l}\text { SERRA } \\
\text { TALHAde Mulungu }\end{array}$ & $\begin{array}{c}\text { Rio Pajéu } \\
\text { Sertão Central }\end{array}$ & $0,00-0,00$ & $0,12-0,14$ \\
\hline
\end{tabular}

*Perda amostral: o município de Buíque não devolveu as amostras de água para a análise laboratorial do flúor.

1 Fonte: Agência Nacional de Águas

2 Fonte: Laudos COMPESA

3 Fonte: Laudos VIGIFLÚOR

Esse fato deve ser considerado porque ressalva a importância de se fazer a fluoretação artificial das águas de abastecimento por parte do poder público. A fluoretação da água é um excelente método preventivo, que atua nos organismos dos indivíduos até mesmo quando estes desconhecem que estão sendo atingidos.

Outro fator relevante é que a fluoretação das águas de abastecimento beneficia proporcionalmente mais a população carente, que não tem acesso a outras fontes de fluoreto para prevenção da cárie, pois seu impacto é maior quanto maior a desigualdade social. Mostrando assim mais um motivo pela qual a fluoretação de águas deve ser feita pelos municípios estudados ${ }^{7}$.

De acordo com o autores ${ }^{2}$ afirmam que a fluoretação das águas deve ser feita, sobretudo em locais onde a prevalência dessa doença é elevada, reafirmando mais uma vez a necessidade de se implementar a fluoretação artificial das águas de abastecimento público.

Os dados fornecidos pelo SISAGUA 2008 mostram que nenhum dos três municípios estudados possui cadastro no sistema sobre o controle e vigilância do teor de flúor nas suas águas. Este dado é de suma relevância porque significa que os municípios não fazem a vigilância dos teores de flúor em suas redes de abastecimento de água tratada, quando as mesmas não contam com a presença ISSN 1982-8829 Tempus, actas de saúde colet, Brasília, 14(1), 77-88, mar, 2020. Epub Mai/2020 
do flúor artificial. Esta atitude impedirá que municípios que eventualmente possuam teores de flúor natural elevado detectem tal elevação, apresentando um risco eminente de fluorose para população consumidora da água de abastecimento.

Segundo os laudos da COMPESA, disponibilizados para essa pesquisa, a presença de flúor natural encontrado nos mananciais que fazem a cobertura do município de Arcoverde, é insignificante no que se refere a prevenção da cárie dentária $(0,01 \mathrm{mgF} / \mathrm{L})$. O laudo do VIGIFLÚOR corrobora com tal resultado pois, $92,66 \%$ da população que recebe água via rede de abastecimento público, consome apenas 0,10-0,20 mgF/L. No município de Serra Talhada, segundo as laudos da COMPESA não foi encontrado flúor natural, com situações parecidas confirmadas nos laudos do VIGIFLÚOR tendo valores sem benefício algum, em relação a cárie dentária, para população $(0,12-0,14 \mathrm{mgF} / \mathrm{L})$.

Os dados obtidos pelo VIGIFLÚOR mostram que os teores encontrados nas torneiras dos dois municípios não contribuem para a proteção da população que consome água da rede de abastecimento público, pois estão abaixo do mínimo ideal, que é $0,55 \mathrm{mgF} / \mathrm{L}$, ou seja, na prática não há benefício no combate à cárie nos municípios estudados.

Entretanto, apesar de não chegar à população que consome a água da rede de abastecimento público, pode ser que existam mananciais com teores de flúor natural em concentração mais elevada que podem estar chegando à população. Essas águas são geralmente encontradas em poços, minas e outras fontes que não possui vigilância pelos órgãos competentes ${ }^{14}$.

No meio urbano e rural é muito comum que os donos de propriedades que não possuem abastecimento da rede pública perfurem poços, geralmente do tipo cacimba, sem autorização prévia dos órgãos competentes e, portanto, sem análise da água, ou envasadoras de garrafões de água que comercializam sem o controle da Agência Pernambucana de Vigilância Sanitária (APEVISA).

Esses motivos elencados justificam a análise periódica das águas ingeridas e, neste caso, em razão do teor de fluoretos naturais, é necessário que a vigilância em saúde dos municípios tenham maior rigor no controle das águas ingeridas de fontes alternativas e também na rede de abastecimento das águas das estações de tratamento, além de um maior rigor por parte da APEVISA das águas envasadas para o consumo humano.

Estudos feitos relataram que as empresas envasadoras de garrafões de água não cumprem com o que determina a legislação, 19\% das amostras analisadas por eles mostraram que os níveis de flúor estavam acima do limite permitido, o que constitui fator de risco para fluorose. Observou-se também discordância entre as concentrações de flúor especificadas no rótulo e as verificadas em análise laboratorial ${ }^{15}$.

\section{CONCLUSÃO}

O estudo demonstrou que os municípios estudados apresentam uma alta cobertura de água 


\section{$87 / /$}

tratada, com exceção de Buíque, o que não significa regularidade do fornecimento. Esse dado é relevante pois, caso os municípios de Arcoverde e Serra Talhada venham a ser fluoretados, uma parte representativa da população seria beneficiada no que diz respeito à prevenção da cárie dentária.

Dessa forma é imprescindível que seja realizado o controle e monitoramento, por parte da vigilância sanitária, dos teores de flúor nas águas de abastecimento público, no caso dos municípios estudados do flúor natural, contribuindo assim para o controle da cárie dentária, bem como para prevenção dos episódios de fluorose.

Diante dos dados obtidos e analisados, esse estudo reforça a necessidade de que os municípios de Arcoverde, Buíque e Serra Talhada tenham suas águas de abastecimento público fluoretadas artificialmente, e o controle de vigilância seja realizado por meio de programas de heterocontrole para atingir seu benefício máximo para proteção da cárie e evitar o risco de fluorose.

\section{REFERÊNCIAS BIBLIOGRÁFICAS}

1. Alves RX, Fernandes GF, Razzolini MT, Frazão P, Marques RA,Narvai PC. Evolução do acesso à água fluoretada no Estado de São Paulo, Brasil: dos anos 1950 à primeira década do século XXI. Caderno de Saúde Pública. v.28. 2008. p.69-80.

2. Ferreira RGLA, Marques RAA, Menezes LMB, Narvai PC. Múltiplos aspectos do uso do flúor em saúde pública na visão de lideranças da área de saúde. Ciênc. Saúde Coletiva. v.18(7). 2013. p. 2139-46.

3. Koppaka R. Ten great public health achievements: United States 1900-1999. US Public Health Service. v.60(19). 2011. p. 619-23.

4. Ramires I, Maia LP, Rizzoli DS, Lauris JRP, Buzalaf MAR. Heterocontrole da fluoretação da água de abastecimento público em Bauru, SP, Brasil. Rev. Saúde Pública. v.40(5). 2006. p.883-9.

5. Cardoso ACC. Participação popular e controle social da fluoretação de águas para consumo humano: um olhar sobre o estado da Bahia. Rev. Baiana Saúde Pública. v. 28(2). 2004 p. 259-66.

6. Brasil. Lei Federal n .6 .050 de 24 de maio de 1974. Dispõe sobre a fluoretação da água em sistemas de abastecimento quando existir estação de tratamento.

7. Frazão P, Soares CCS, Fernandes GF, Marques RAA, Narvai CP. Fluoretação da água e insuficiências no sistema de informação da política de vigilância à saúde. RevAssoc Paul Cir Dent. v. 67 (2). 2013. p. 94-100.

8. Pucca J A. Política Nacional de saúde bucal como demanda social. Ciênc. Saúde Coletiva. ISSN 1982-8829 Tempus, actas de saúde colet, Brasília, 14(1), 77-88, mar, 2020. Epub Mai/2020 
v. 11(1). 2005. p.243-6.

9. Brasil. Ministério da Saúde. Secretaria de Atenção à Saúde. Departamento de Atenção Básica. Coordenação Nacional de Saúde Bucal. Diretrizes da Política Nacional de Saúde Bucal. Brasília (DF): Ministério da Saúde. 2004.

10. Saúde, da Ministério. Guia de Recomedações para o uso de Fluoretos no Brasil, 1ed. Brasília - DF, 2009.

11. CECOL/USP. Centro Colaborador do Ministério da Saúde em Vigilância da Saúde Bucal. Consenso técnico sobre classificação de águas de abastecimento público segundo o teor de flúor. São Paulo: Faculdade de Saúde Pública da Universidade de São Paulo. 2011.

12. BleicherL, Frota FHS. Fluoretação da água: uma questão de política pública - o caso do Estado do Ceará. Ciência \& Saúde. v.11(1). 2006. p. 71-8.

13. Wambier DS, Pinto MHB, Kloth AEG, Vetorazzi ML, Ditterich RG, Oliveira DK. Análise do teor de flúor nas águas de abastecimento público de Ponta Grossa-PR: dez meses de heterocontrole. Biol. Health Sci. v.13(1/2). 2007. p.65-72.

14. Lima FG, Lund RG, Justino LM, Demarco FF, Del PFAB, Ferreira R. Vinte e quatro meses de heterocontrole da fluoretação das águas de abastecimento público de Pelotas, Rio Grande do Sul, Brasil. Cad. Saúde Pública. v.20(2). 2004. p.422-29.

15. Grec RHC, Moura PG, Pessan JP, Ramires I, Costa B, Buzalaf MAR. Concentração de flúor em águas engarrafadas comercializadas no município de São Paulo. Ver. Saúde Públ. v. 42(1). 2008. p.154-7.

16. Guia nacional de coleta e preservação de amostras. Água, sedimento, comunidades aquáticas e efluentes líquidos. Brasília-DF, 2011.

17. Instituto Brasileiro de Geografia e Estatística. http://www.ibge.gov.br/

18. Sistema Nacional de Informações sobre Saneamentohttp://www.snis.gov.br/

Artigo apresentado em outubro de 2019 Artigo aprovado em janeiro de 2020 Artigo publicado em maio de 2020 